\title{
Practicing strategic metadata management: Knowing your media and its market potential
}

\section{Cindy Cunningham}

worked as a journalist in California and Washington State before getting her library degree at the University of Washington. She has worked at the Library of Congress, University of Washington, and Kitsap Regional Library before being recruited by Amazon.com where she worked for six years. She has been Director of Cataloging at Corbis Corporation since February 2004.

Keywords: controlled vocabulary, image cataloging, search and metadata, image market potential, thesaurus, DAM management

Abstract Managing metadata for a digital asset management (DAM) system can be most effectively done if the DAM manager considers the market potential of the media and takes customer needs, company objectives and industry practices into consideration when assigning appropriate metadata. The DAM manager should also guarantee search success for an image or other media object by developing a thesaurus, applying controlled vocabulary and leveraging the underlying data structure.

Cindy Altick Cunningham Director, Cataloging Corbis Corporation 710 Second Avenue Suite 200

Seattle, WA 98104 , USA Tel: +1 $206373-6260$ Fax: +1 206 373-6100 Email: cindyc@corbis.com
To the manager of a digital asset management (DAM) system, the need for metadata can quickly become obvious, yet collecting and creating the right metadata can be overwhelming. Metadata - that general word used to describe all the text that can accompany an image, from the date created and photographer name to subject categories, are not all the same. The critical information is clear, like making sure a creator's name and appropriate rights and copyright information are attached to every media object. Some kind of text is necessary for successful long-term storage - does one use dates, locations, personal names, or even a classification system to keep track of past images or documents? Deciding which metadata to use to file a media collection is a many faceted challenge.
Understanding the media's marketability can give a DAM manager a flexible way to manage media and its metadata.

Which metadata are important depends upon the intention and possible market for the media. For example, if a photographer takes great shots of a remote mountain range, but fails to note the names of the specific peaks or the direction of the shot (looking east) and possibly outstanding features of that peak - glaciers, faces that are often climbed etc - the image will not be usable by the outdoor and climbing magazine market. If the photographer thought the picture was a gorgeous mountain at sunset picture, and that it could be used by the commercial market, it may work without any of these specifics. But even the commercial market may want to know where the 
shot was taken, for well recognized peaks will be known to the audience seeing the picture and may be part of the gimmick or point of the ad. The photographer in this example, therefore, must understand the marketplace and potential for the image he is creating. Similarly anyone in charge of a media collection must have a good idea of the consumers for the media and the how to make media as versatile as possible for market potential.

Applying metadata to media is a costly process. As head of a cataloging team at Corbis Corporation, one of the largest image companies in the world, I am constantly working with my team to hit that perfect balance between wellcataloged and cost-efficiently cataloged images. Corbis began its collection by acquiring digital images of da Vinci, and went on to acquire images of Vermeer, Klimt, Hopper and other major artists. Owning such works demands specialists with an art history background. When Corbis acquired the Bettman Archive in the mid-1990s, it committed itself to having specialists who could catalog both the well-known but also the obscure historical images. Without adequate source text, the cataloging process can take anywhere from ten minutes to an hour. My team tries to identify specific locations, notable buildings and works of art, people depicted, historical time period, a date and of course a creator - photographer, illustrator artist, etc. The challenge is to understand the market for each image and to apply metadata as needed. Hitting the balance between describing the image well enough and "over cataloging" can be a fine line with some of our images. Many of our fine art and historic images end up being used for commercial purposes, where the specifics of the image may never need to be identified. However, Corbis also has a huge publishing market - and magazine and book publishers want to know all the details of an image before they will purchase it. Knowing how much needs to be present on an image to attract a customer, and how much possible further research we can do later without losing the sale is an evolving science.

Ideally all metadata mangers will develop good relationships with those creating the media so as to get reliable metadata from them. Some companies post detailed requirements for image providers to follow regarding the metadata. If companies are receiving images from outside their database they expect the image providers to understand the possible marketing potential of what they are providing and to supply the correct words. Similarly, if a media collection is entirely created and managed internally, it is the role of the DAM manager to understand the potential of the collection and where the company is going with the collection, so as to develop an appropriate system of classification and attribute assignment and to know what else needs to be added to the media to further its usability.

Aside from considering the marketability issues and judging media by its possible customers, there is also the consideration of search and retrieval. For example, when all images are arranged in a database, and the only access to these images is via words, it is very challenging to meet the needs of all searchers. Many people using an image database are visually oriented and may or may not have an extensive and varied 
vocabulary. Further, they may only have an idea - not a specific image in mind. The searcher will want to brainstorm on the idea of "family" or "happiness," or "success" or "living well." The searcher hasn't settled on an image and needs to be able to throw conceptual terms into the database and see some ideas. From there a single image may spark an idea, and their next search will be literal and specific - like "car, winding road, desert." Given the brainstorming nature of much creative work - and the pre-internet practice creatives had of thumbing through catalogs to get ideas - some general, conceptual access to images is necessary to accommodate this audience.

Understanding the types of vocabularies of customers is an important part of creating a thesaurus of searchable terms. There are many words a customer might use to describe something jumping, jump, woman, female, girl, joy, happiness. A well-developed database, designed to support exhaustive searches, will use controlled vocabulary along with lead-in terms, related terms and broader and narrower terms, so as to capture variant verb forms and categories. Is joy different from happiness or elation? Should one lead to another? How many fine distinctions between concepts do you want to create, and how sure do you want to be that your searcher never gets zero results?

Controlled terms can also guarantee that every time a user types in "Bill Clinton" images of the former president will be returned, even if some of them were originally identified as William J. Clinton, President Clinton or any other variant of his name. News images in particular can be guilty of presenting many variant spellings of names and places in the caption and location fields. Applying some controlled keywords in addition to titles and captions that bring all like images together under a common term, is critical to creating successful search results.

In the early days of online databases I used to teach librarians that in order to search a database successfully, one had to know all one could about the nature of the database itself. In those early days there were often scope notes available that told the users what was in the database - years covered, if they were articles or citations, books or images, and how the data was structured. For example, the notes would tell you if there was considerable text in a caption field, so you could use a proximity operation, like "with" to indicate that the words you were searching should be within the same sentence. Obviously it would help to know if you had that option, or if your database was simply comprised of keywords, where proximity would have no impact.

Searching has become easier, and a more public exercise - users do it for themselves and don't count on an information specialist or a librarian to do the search for them. Furthermore, with the advent of Google, people don't feel the need to understand anything about the underlying data; they assume they can put a word in a search box, and something relevant will be returned. Of course it's not that simple, though modern search technology is doing its best to anticipate who the user is using cookies and past search behavior, IP addresses and email addresses - and what the searcher likely needs. In this new world the computer is trying to be very smart so that the user can be uninformed. 
However, the search experience is still only as good as the metadata that can be searched, and media being searched using an electronic database fare best when they are described for every possible market and for every possible user and user style.

As I mentioned earlier, cataloging is expensive. It takes time to research an image, to consider all facets of it that need description, and obviously a cataloging department must make many decisions about how large and how structured its controlled vocabulary is going to be, with related terms or broader or narrower terms. Making decisions about how to create a usable and reliable thesaurus of terms while leveraging the data structure is dependent upon an understanding of recall and precision, the two pillars of searching. One can only choose to have better recall (more results) or better precision (more precise results). The two live in a constant balance, and one can never have both at the same time. A DAM manager who understands this can design a vocabulary and search method to assist searching along one or the other of these axes, by either creating a detailed and specific vocabulary with few broader terms, or a general set of terms leading to broader and related terms.

All of this leads us back to the dilemma of the DAM manager overseeing a collection and making sure that each image or object's full marketing potential is understood, so that appropriate metadata can be applied. We must remember that in the online world an image doesn't have to have a single identifier; it can have many, seemingly conflicting attributes. In the photography world this translates into describing some locations generically if they could be applied to several possible uses - as a Florida or a Caribbean resort image, for example or as a South American, Caribbean or European person of color. While in the literal world of cataloging one would want to make a decision about the accuracy of describing the image - is the person in Miami - would we describe that person as Black South American or Indian? In this scenario all possible ways of interpreting the image can be applied as attributes, so that someone looking for an image of a tropical scene could find any image that might apply.

When I worked at Amazon.com in the early days, this realization, that a book can live in more than one place, was just hitting the publishing market. The classification system it used to place books on the floor of a bricks and mortar bookstore was expandable in the internet world - a publisher marketing a book didn't have to decide if the book was biography or history; it could be both. Besides making this leap into the virtual world, publishers had to understand in general that the metadata about the book in an online bookstore was the equivalent of the physical book in a bricks and mortar bookstore. It was a constant struggle in the early days to get accurate information about books that we were selling. Publishers weren't well organized enough to send out extensive written information about the book, let alone scans of the cover, back page or inside illustrations. Even though libraries, with their card catalogs, had been around for hundreds of years, the notion of a block of text being the equivalent of the actual object in an online bookstore took some getting used to. 
All of us who manage DAM must take the same approach, in understanding that no matter how strong the images might be or how interesting the media, the only and most obvious access points are the words used to describe and categorize the object. Without good metadata, the media is invisible to the world and may never be found. 